\title{
Cultural Heritage Destruction: Documenting Parchment Degradation via Multispectral Imaging
}

\author{
Alejandro Giacometti \\ Dept. of Medical Physics \\ Centre for Digital Humanities \\ University College London \\ alejandro.giacometti.09@ucl.ac.uk \\ Simon Mahony \\ Centre for Digital Humanities \\ University College London \\ s.mahony@ucl.ac.uk \\ Tim Weyrich \\ Dept. of Computer Science \\ University College London \\ t.weyrich@cs.ucl.ac.uk
}

\author{
Alberto Campagnolo \\ Ligatus Research Centre \\ University of the Arts, London \\ a.campagnolo1@camberwell.arts.ac.uk
}

\author{
Melissa Terras \\ Centre for Digital Humanities \\ University College London \\ m.terras@ucl.ac.uk
}

Adam Gibson

Dept. of Medical Physics

University College London

agibson@medphys.ucl.ac.uk

\author{
Lindsay MacDonald \\ Photogrammetry, 3D Imaging \& \\ Metrology Research Centre \\ University College London \\ ucfs/wm@ucl.ac.uk \\ Stuart Robson \\ Photogrammetry, 3D Imaging \& \\ Metrology Research Centre \\ University College London \\ s.robson@ucl.ac.uk
}

\begin{abstract}
This paper describes the methodology and presents preliminary results of a project using multispectral imaging to document the deterioration of parchment. A series of treatments has been applied to degrade samples from a deaccessioned manuscript using both physical and chemical agents. Each sample is photographed before and after the treatment by a multispectral imaging system to record the effect of the treatments on both the writing and the parchment. Here we present the initial imaging of the samples, and we show how some of the agents affect the writing and parchment.
\end{abstract}

Multispectral imaging. Manuscript. Parchment degradation.

\section{INTRODUCTION}

Digital surrogates of documents have become an indispensable tool for scholars and archives. They provide an easily accessible, non-degrading resource for research and study (Terras \& Robertson, 2006). Numerous imaging and image processing technologies have been employed to examine the characteristics of the originals, such as X-ray imaging, imaging in the infrared spectrum, and multi-spectral imaging. These offer additional information about the physical characteristics and condition of the document. The results have been better images, more possibilities for analysis, and additional information recovered from ancient documents. (Chabries, Booras, \& Bearman, 2003; Salerno, Tonazzini, \& Bedini, 2007; Tanner \& Bearman, 2009).

Digitisation projects generally concentrate on documents in their existing state. Typically the focus of image processing is to restore the digitally rendered document to its supposed original state, before the damage occurred. In this project, we aim to investigate the processes of controlled document degradation using multispectral imaging. We have obtained a manuscript on parchment, which we are subjecting to a variety of degradation procedures. We are documenting the process via multispectral imaging. In this paper, we present the programme, give an outline of the methodology for degrading the manuscript, and describe the approach to imaging. Finally, we show some samples from the initial imaging trials, and preliminary results from our tests with the various degradation methods.

We obtained an $18^{\text {th }}$-century manuscript deaccessioned from the London Metropolitan Archives. Although it was in good physical condition, this manuscript was deemed to hold no historical value, and it was kindly provided to us for experimentation. It comprised two large sheets of prepared animal skin, i.e. parchment, written in iron gall ink and highlighted in red ink. Each sheet 
measured approximately $70 \times 70 \mathrm{~cm}$ (Figs. 1 and 2), and the two had been tightly folded together for storage. It was dated $11^{\text {th }}$ August 1753 , in the reign of George II, and appeared to be an indenture (contract) between a Mr John Sherman and Mr Christ Gardiner.

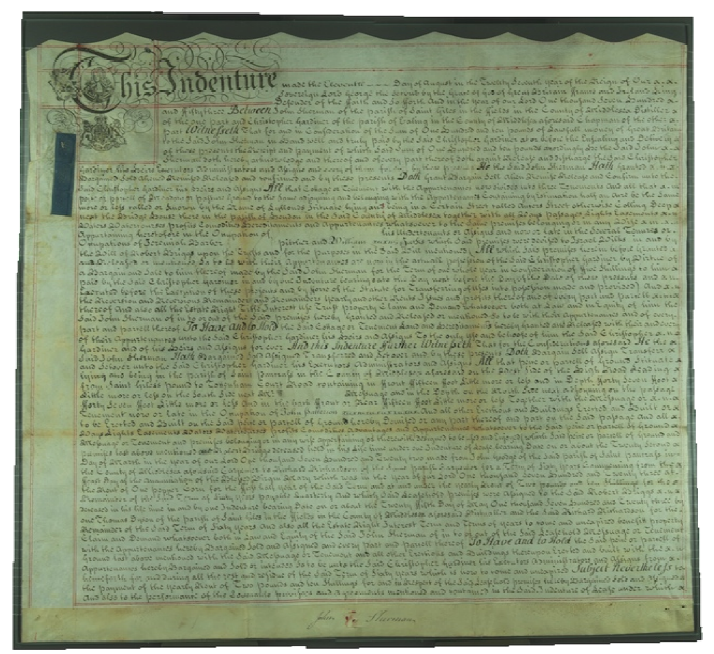

Figure 1: Outer sheet of the complete manuscript, showing writing in Iron Gall ink on parchment



Figure 2: Inner sheet of the complete manuscript

\section{MULTISPECTRAL IMAGING}

A multispectral image is a set of images acquired through narrow band filters for consecutive wavebands. Each image shows the intensity of radiation from the scene in the corresponding waveband (Vane \& Goetz, 1988). Images are acquired at visible wave-lengths $(400-700 \mathrm{~nm})$, and may also include regions of the non-visible spectrum: ultraviolet $(<400 \mathrm{~nm})$ and infrared $(>700$ $\mathrm{nm}$ ). Images that include more channels over a broader range of wavelengths are referred to as hyper-spectral images (Landgrebe \& Chen, 1999). Multispectral imaging was developed for remote sensing to determine the composition of objects in space by measuring their emission spectra. NASA satellites were also designed to capture multispectral reflective images from Earth, the most famous being Landsat, first launched in 1972, with eight spectral bands. The original purpose was to identify the kinds of resources that could be found in a specific location without the need for high spatial resolution. The idea was to analyse spectral variations in a pixel rather than the spatial variations in higher resolution images (Landgrebe \& Chen, 1999).

Multispectral imaging (MSI) has been successfully applied to the imaging of fine art paintings (Hardeberg, 2001). It has also been utilised to image ancient documents (Attas, 2004; Salerno et al., 2007). Bearman and Spiro (1996) were the first to use digital MSI on the Dead Sea Scrolls, with a liquid crystal tunable filter in front of a monochrome CCD camera. They captured digital images from 400 to $1050 \mathrm{~nm}$, and noted the great improvement in legibility at $970 \mathrm{~nm}$, compared with the image at $700 \mathrm{~nm}$.

Multispectral images can be used to identify, and hence to separate, different types of inks in a single document, allowing a researcher to determine whether the document has been edited, or if it was written by several different hands. Additionally, the reflectance profiles of known materials can be stored in a database and used to classify unknown materials. Recognising the type of ink can help determine the age or location of a specific writing.

The type of ink used has considerable impact on the imaging approach. The earlier carbon black ink consisted of graphite or soot particles suspended in an organic binder and applied with a stylus. These inks do not penetrate or stain the parchment but rest on top, with particles adhering to the microstructure of the surface. Iron gall ink, also known as gallo-tannin ink, was introduced around the third century $A D$, prepared with organic material that penetrates the substrate more deeply and reacts to dye it black. Chabries et al (2003) noted that the parchment reflectance increases at longer wavelengths, resulting in greater contrast of text in the infrared images.

\section{METHODS}

From the original manuscript, 23 flat square sections of $8 \times 8 \mathrm{~cm}$ were cut, with each section containing written text. Two sections have text written on both sides. Each sample will be exposed to an external deteriorating agent in order to force a rapid and controlled form of degradation. The agents include mechanical damage, heat, humidity, abrasion and a variety of substances with different chemical properties (Table 1). Each will affect the appearance and condition of the samples in 
different ways, typical of the actual damage suffered by parchment in real archives. In the following sections we describe the methods for degrading the parchment and for imaging.

\subsection{Degradation treatments}

Large collections of parchment documents exist in public and private libraries, archives, and museums in varying degrees of preservation. Parchment has a predominantly organic composition, mainly based on protein collagen in association with small quantities of elastin, globular proteins, and fats. Parchment is prepared from a pelt, i.e. an animal skin that has been wetted, immersed in lime water (a saturated solution of Calcium Hydroxide), dehaired, and scraped. The pelt is then left to dry under tension on a wooden frame. The stretching of the soaked pelt has the effect of reorganizing the collagen fibre network into a laminate structure, and, as the pelt dries, the fibres are locked into the stretched condition. The resulting material is a fairly stiff sheet which, without any further treatment, is durable and can last for centuries, provided it is kept cool and dry (Reed, 1972; Clarkson, 1992; Larsen, 2007).

Parchment is an extremely hygroscopic material that reacts readily to changes of humidity with changes in thickness and physical appearance, such as cockling and curling. It is also endangered by biological, thermochemical, and mechanical degradation.

We have selected some methods of artificial degradation for our parchment samples to simulate the kinds of damage that can affect parchment documents found in cultural heritage collections. These degradation methods fall within the main categories of mechanical, thermochemical and humidity, biological, and physical damage. These act directly upon the writing medium - i.e. the parchment - changing its physical characteristics at both microscopic and macroscopic levels, as well as damage caused by external substances that act directly to obscure the text.

Table 1: Methods for degrading the parchment

No. Treatment Description

1. Control Control parchment from inner sheet sheet, no treatment.

2. Control Control parchment from outer sheet sheet, no treatment.

3. Fold Control parchment containing a control fold-no treatment. Fibres at the fold are more degraded than fibres from other parts of the document due to their exposure to physical and mechanical damage and dirt.

4. Ultra-

Expose the parchment to violet ultraviolet light. The energy in light photons can cause intermolecular and extra-molecular cross-linking in the collagen fibres. When this occurs, at a microscopic level the collagen fibres will shorten, fray, and flatten; at a microscopic level prolonged exposure to light will eventually lead to discoloration, fading, and fragility. The process is slow, but cumulative.

5. Mould Keep parchment in an enclosed high-humidity (>90\%RH) environment for an extended period of time until mould growth is detected. The parchment is subsequently desiccated. Mould grows on documents in humid (>75\%RH) and warm (>22-25C) conditions. As microorganisms grow and feed on the parchment, multiple effects can be detected. At the microscopic level the collagen fibres appear split and shortened; at the microscopic level the appearance of the medium is altered, with changes in colour and mass - e.g. thinning. (Součková, 2007).

6. Scraping Forcibly remove the surface layer and the visible writing off the parchment through the use of pumice stone. Scraping was a common way of deleting, and correcting text on parchment, as well as a way to reuse the medium for a completely new text - e.g. palimpsest documents.

7. Mech- Alter the surface of the anical parchment via double folding and damage rolling. The surface of parchment document can be affected by wear and tear. This is usually most notable in the first and last membranes of a codex, especially if the binding has partially or totally failed. The text can become difficult to read, and the surface of the medium becomes softer and velvety as the grain and fibres are broken by the repeated mechanical action.

8. Scrunching Alter the surface of parchment by scrunching. Badly stored documents, especially single-leaf ones not in codex or scroll form, or pages that have become detached, can become crumpled, altering the physical appearance of the medium. The writing inks, especially on a degraded parchment, can flake away under these conditions; miniatures and other coloured portions of text are particularly susceptible to this 
9. Heat

kind of damage.

Submit the parchment to temperatures above 200 C. Dry heat removes most of the water from the collagen structure and the molecules are thus closer together and cross-linking occurs more frequently and freely under these circumstances. Collagen fibres will shrink, flatten, and split, while at the microscopic level the document will undergo quite drastic changes in dimensions. (Vest et al., 2007).

10. Smoking Apply smoke to the parchment. Smoke temperatures are kept low in order to separate the effect of smoking and heat. Smoke alters the colour of the medium, while soot obscures writing further.

11. Iron gall

12. India ink

13. Black tea

14. Wine

15. Blood
Stain the parchment with modern iron gall ink. Sometimes a text was corrected, or altered, at a later stage, by obscuring letters or words deemed wrong with ink. metallogallic based ink recipes varied greatly from person to person, and period to period.

Stain the parchment with India ink. India ink is composed of lampblack, combined with water and a binding agent like gelatine, shellac, or gum Arabic. Carbonbased inks were amongst the first kind of writing inks used. Their chemical composition and the physical characteristics of their dried form is very different from those of metallogallic inks.

Stain the parchment with black tea. Black tea is rich in tannins. Tannins have marked astringent properties and draw collagen fibres closer together, reducing the water content of the skin. Vegetable tannins have also a colouration effect, mainly shades of reds and yellows. The effect of this colouration would not wash away once set. (Reed, 1972).

Stain the parchment with red wine. Wine is also rich in tannins, but the colouring effect is less conspicuous and the tanning effect is less prominent, which will influence spectral response.

Stain the parchment with animal blood. Blood stains can occur in historical documents. These appear as brownish stains that can sometimes obscure the text. Unlike paper documents, parchment cannot be washed during conservation and stains are thus not usually removable. Moreover, blood looks similar to iron gall ink as haemoglobin is rich in iron.

16. Aniline Stain the parchment with aniline dye dye. Although fairly modern - it was first isolated in 1826 and used in the dye industry since the 1850 s - this chemical compound is used to dye leather. Colouration of skin by aniline dyes is permanent, making reading the text very difficult if not impossible to the naked eye. Chemically, aniline dyes are very different to iron gall ink.

17. Oil

Stain the parchment with an oily or greasy substance. Oily stains are common in historical collections. Oil replaces the air trapped inside the skin and makes the parchment translucent. If a document contains text on both recto and verso, both writings will be visible from both sides making reading difficult.

18. Desiccant Enclose the parchment in an airtight container with desiccant for a period of time, until the humidity drops below $30 \% \mathrm{RH}$. When parchment is stored in a very dry environment, irreversible cross-linking of the collagen structure can take place as the collagen molecules are in close contact in the absence of intermolecular water content. (Vest et al., 2007).

19. Sulphuric Apply Sulphuric acid $\left(\mathrm{H}_{2} \mathrm{SO}_{4}\right)$ to acid the parchment. The concentration of acid is kept low in order to observe the changes, while maintaining its structural integrity. Beginning in the early 19th century, sulphur compounds were used to speed up the process of preparing the pelt. These sulphur compounds remain in skin even after rinsing and eventually convert into sulphuric acid. Skin treated in this fashion is weaker and greyish in appearance.(Reed, 1972)

20. Hydro- Apply Hydrochloric acid (HCl) to chloric the parchment. The concentration acid of acid is kept low in order to observe the changes, while maintaining its structural integrity. Non-oxidising acids like hydrochloric were used in production of iron gall inks. Although parchment is more resilient to acidic (and alkaline) substances than paper, an excess of acidity in ink 
can perforate the parchment (Banik, 1997).

21. Calcium Apply calcium hydroxide hydroxide $\left(\mathrm{Ca}(\mathrm{OH})_{2}\right)$ to parchment. Calcium hydroxide is a strongly alkaline substance, used in the production of parchment as a dehairing and defattening agent.

(Reed, 1972)

\subsection{Multispectral imaging}

Each sample is imaged before and after the treatment, using two different cameras, in combination with two lighting systems for both reflective and transmissive imaging, under a range of bandpass filters. The cameras are: (1) Nikon D200 with $105 \mathrm{~mm}$ lens, an RGB digital SLR, which captures images in the visible spectrum; (2) Kodak Megaplus $1.6 \mathrm{i}$ scientific monochrome camera, which captures images in both the visible and nearinfrared spectrum over the range 400-1000 nm. With each camera, a set of bandpass optical filters centred at regular intervals is used.

Each of the first 16 filters has a bandwidth of approximately $20 \mathrm{~nm}$, at wavelength intervals of 20 $\mathrm{nm}$ throughout the visible spectrum from 400 to 700 $\mathrm{nm}$ inclusive (Fig. 3). The last 5 filters have a bandwidth of approximately $50 \mathrm{~nm}$, at intervals of $50 \mathrm{~nm}$ in the near-infrared spectrum from 750 to $950 \mathrm{~nm}$ inclusive.

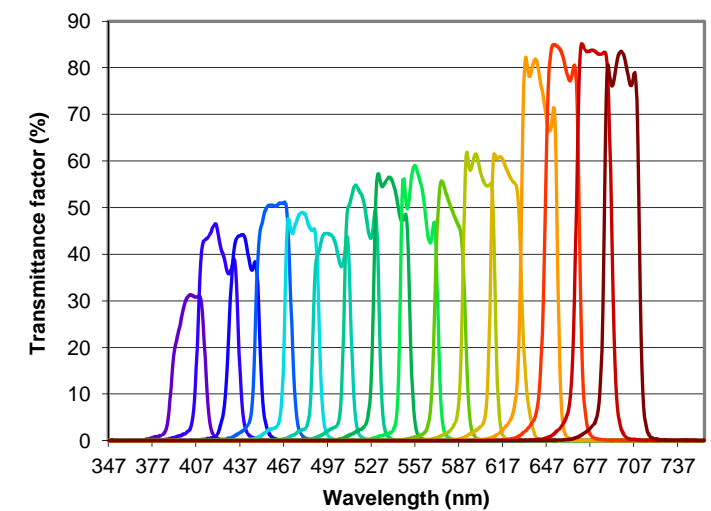

Figure 3: Transmittance factors of 16 optical bandpass filters in the visible spectrum

We use two lighting systems. The sample is illuminated at $45^{\circ}$ angles by a set of four tungsten halogen lamps in a standard photographic document copystand. This setup allows the camera to capture light reflected from the parchment without specular highlights. The second system uses a lightbox with two fluorescent lamps and a diffuser to illuminate the parchment from behind, thus capturing the transmitted light.
At the end of this project, there will be a total of 3900 images, captured in 2 sessions, in 4 modalities (Table 2). There are 23 square parchment samples in total, but two samples contain written text on two sides. These samples are imaged once on each side.

Table 2: Imaging details

$\begin{array}{lccc}\text { Modality } & \text { Squares } & \text { Filters } & \text { Session } \\ \text { RGB Reflective } & 25 & 17 & 425 \\ \text { RGB Transmissive } & 25 & 17 & 425 \\ \text { Monochrome Reflective } & 25 & 22 & 550 \\ \text { Mono. Transmissive } & 25 & 22 & 550 \\ \text { Session Total } & & & 1950 \\ \text { Total (2 Sessions) } & & & 3900\end{array}$

We punched four $1 \mathrm{~mm}$ round holes in each sample at about a third of the distance from the borders. These holes are apparent in each image and will be a persistent feature after the samples are degraded, both as reference points for registration of the multispectral image channels and for comparison of image sets of the samples before and after treatment.

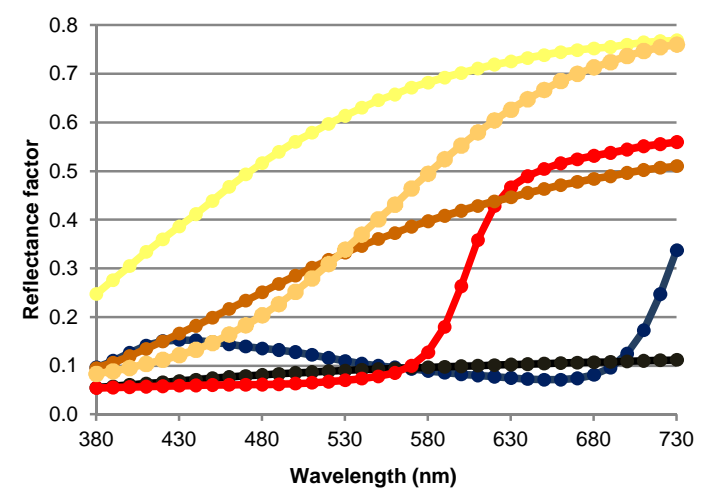

Figure 4: Measured reflectance spectra of (top to bottom) parchment, yellowed parchment, dirty parchment, red ink, blue seal, and iron gall ink

As a reference for reconstruction of the reflectance spectra from the multispectral image sets, we imaged a Mini-Macbeth colour checker chart using an X-Rite i1Pro spectrophotometer. This instrument measures the reflectance factor of each patch at $10 \mathrm{~nm}$ intervals from 380 to $730 \mathrm{~nm}$. Typical spectra from the manuscript are shown in Fig. 4, also measured by the i1Pro. The parchment has a very smooth spectrum, lower at the blue end, giving its characteristic yellow hue. The loss of reflectance at short wavelengths is accentuated in the 'yellowed' regions. At a middle wavelength of $550 \mathrm{~nm}$ the reflectances of the ink and parchment are 0.1 and 0.65 respectively, giving a contrast ratio of $\sim 6.5: 1$.

\section{INITIAL IMAGING TRIALS}


We imaged the parchment under a glass sheet to hold it flat, using the Nikon RGB camera. Each sample was first imaged without a filter on the camera (Fig. 5). The four reference holes can be observed as round black spots.

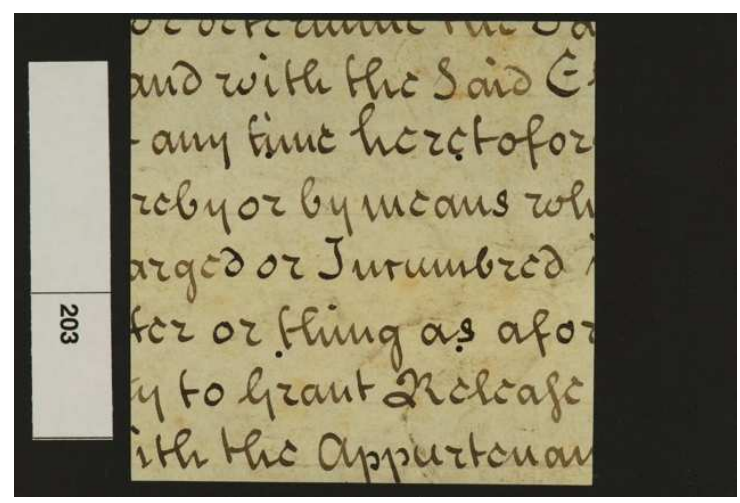

Figure 5: Full spectrum reflective $R G B$ image of parchment sample 203

We then captured each sample 16 times, through successive filters (Fig. 6). The first and last images are completely dark, due to the low sensitivity of the camera sensor at these wavelengths. The progression of hues, from blue through green to red, matches the perception of those wavelengths by the human eye. Note that the second image also has a hint of red, because the red channel of the camera is designed to be somewhat sensitive to these short wavelengths in order to be able to differentiate the violet hues of the spectrum.

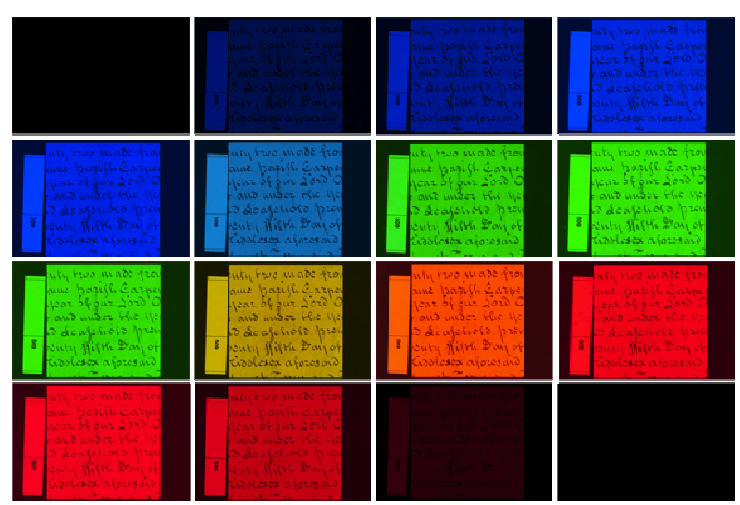

Figure 6: Reflective RGB multispectral image set of sample 509 at 16 wavelengths from 400 to $700 \mathrm{~nm}$

Each sample was also imaged using the transmissive lighting system (Fig. 7). Sample 602 has text on both recto and verso sides. In the images under transmissive light, the two layers of text can be observed. The reference holes in these images appear as bright spots, rather than dark ones, because the light is coming from beneath the parchment.

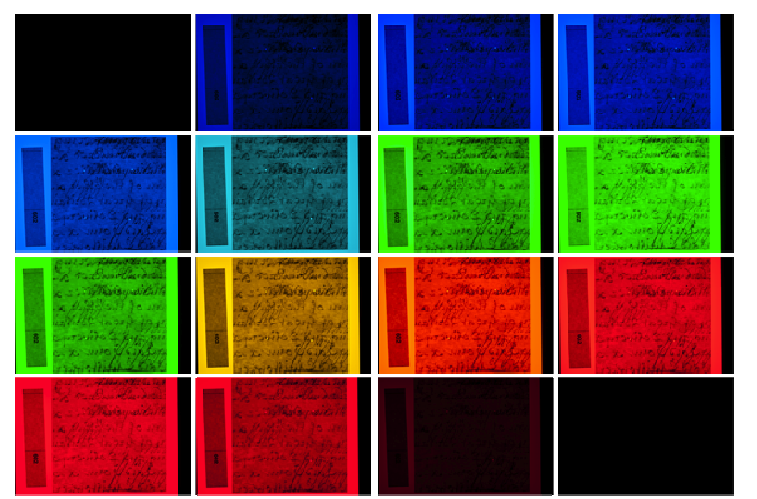

Figure 7: Transmissive RGB multispectral image set of parchment sample 602, recto side

\section{DEGRADATION TESTS}

Preliminary tests on the degradation methods, using 'spare' pieces of parchment, illustrate various effects on both the appearance and physical characteristics of the parchment. Figure 8 shows the difference between the effects of scraping, scrunching and mechanical damage. The scraping appears to have removed almost all traces of ink from the surface. Mechanical damage is also effective in deteriorating the writing.

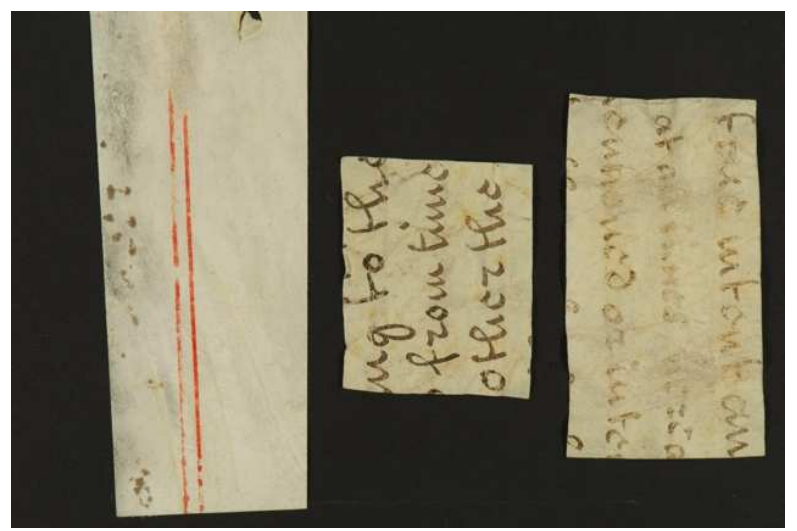

Figure 8: Three test samples submitted to scraping, scrunching and mechanical damage, imaged under reflective lighting

Samples were stained with India ink, iron gall ink and aniline dye (Fig. 9). Under natural light, the appearance of iron gall ink and India ink is indistinguishable. However, they differ in both tone and intensity from the original iron gall writing. The aniline dye completely obscures the writing.

Oil, tea and red wine affect the parchment in quite different ways (Fig. 10). The oil makes the substrate more transparent, causing the writing on the two sides to blend together. Tea, on the other hand, seems to have reactivated the original iron gall ink and made it bleed. Red wine appears to have deeply stained the parchment, and hidden the writing. 


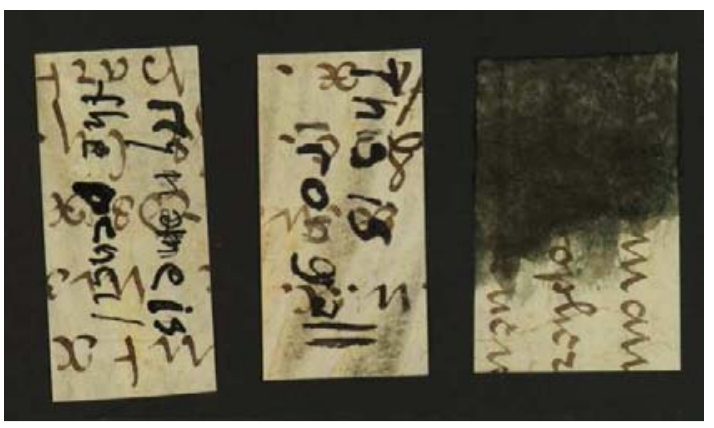

Figure 9: Three test samples stained with India ink, iron gall and aniline dye, under reflective lighting

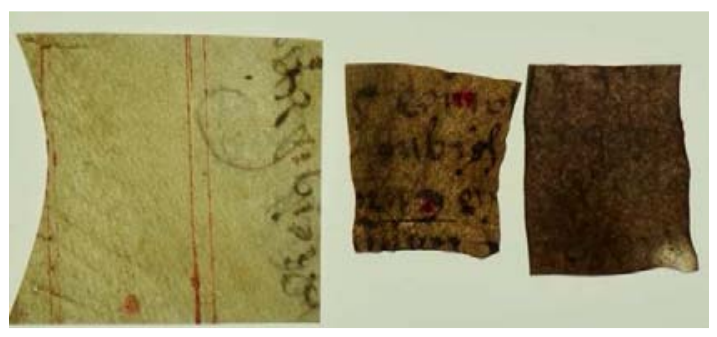

Figure 10: Three test samples submitted to soaking in oil, tea and red wine, in transmissive lighting

When the same samples are imaged under reflective lighting, however, the writing on the sample treated with wine is perfectly readable (Fig. 11) and the effect of oil is less noticeable.

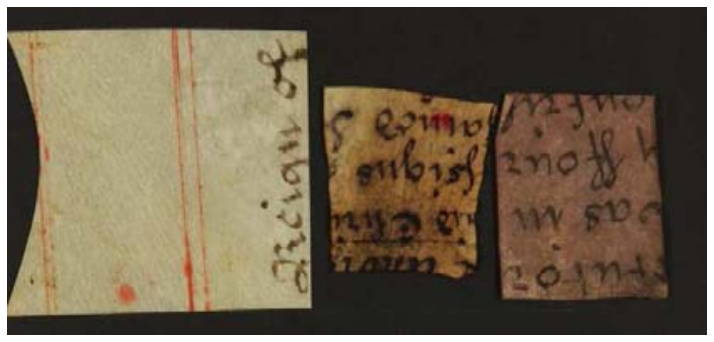

Figure 11: Three test samples submitted to soaking in oil, tea and wine, under reflective lighting

The colour and transmittance of parchment can vary significantly with the degradation methods (Fig. 12). The desiccated sample has become lighter, while the smoke has turned parchment deep yellow. The blood has made the parchment red like meat, and obscured the writing to imaging under both transmissive and reflective lighting (Fig. 13). Burning has made the parchment very dark and opaque, and shrunk it to half the original size.

The burnt sample, moreover, has become extremely fragile, and cracked in the middle. Reflective lighting reveals the writing on the burnt sample, especially at infrared wavelengths. Under a much longer exposure with backlighting the burnt sample does allow some light through, and delivers some contrast between the parchment and ink (Fig. 14).



Figure 12: Four test samples submitted to desiccation, smoking, soaking in blood and burning, imaged under transmissive lighting

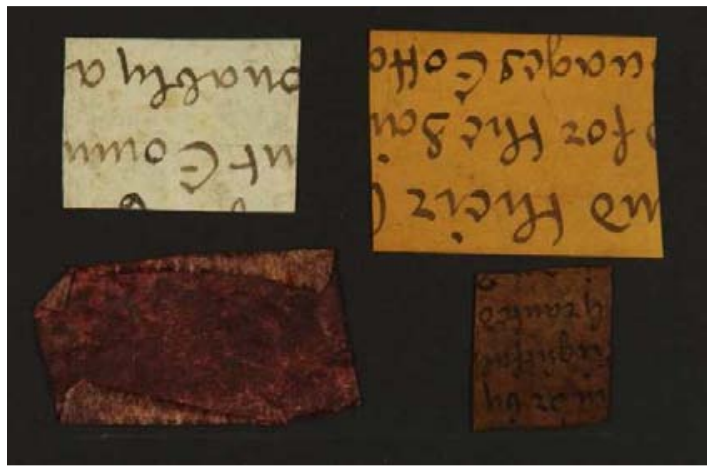

Figure 13: Four test samples submitted to desiccation, smoking, soaking in blood and burning, imaged under reflective lighting

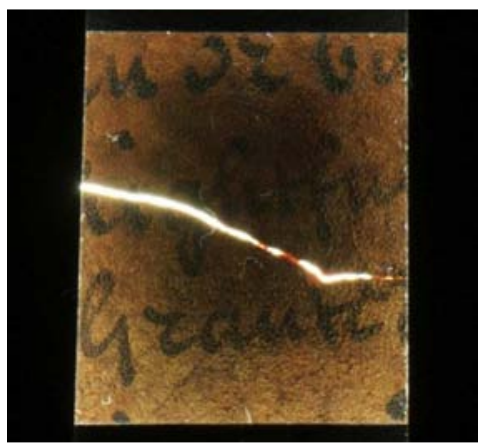

Figure 14: A burnt test sample, imaged through transmissive lighting using a very long exposure

\section{DISCUSION}

At the completion of this project we will have a set of multispectral images showing both the initial and degraded state of a manuscript. These images will be fully documented and released publicly as a resource for the research community. They will provide insight into how a parchment with text reacts visually and physically to various forms of degradation. We offer these as a guide for 
conservation, a resource for quantitative evaluation of image processing algorithms for information recovery, and for other research activities in conservation, image processing, computer graphics, and archaeometry.

We have established a methodology for a comprehensive study of how the degradation of parchment affects its appearance, changes its physical characteristics, and influences our ability to discern written text. Additionally, we have presented an overview of how this degradation will be documented using a multispectral imaging system.

We have shown examples of how the effects of degradation treatment to parchment can vary, and how multispectral imaging analysis can be instrumental in studying these effects.

Analysis of multispectral images of scraped or mechanically damaged samples will enable us to identify even faint traces of ink that might not be visible. It will enable the identification of seemingly identical inks, and could allow us to recover writing from darkly stained parchment, identifying the staining agent, and from charred and burned fragments.

The data from our experiment will be made available to the public. Our dataset of the parchment samples before treatment will provide a 'ground truth' for analysis of the effectiveness of image processing algorithms in restoring images of the degraded samples.

\section{REFERENCES}

Attas, E. (2004). Enhancement of Document Legibility Using Spectroscopic Imaging. Archivaria, 57, 131-146.

Banik, G. (1997). Decay caused by Iron-Gall Inks. In Iron Gall Ink Corrosion. Iron Gall Ink Corrosion Symposium. Rotterdam; Amsterdam: Museum Boijmans van Beuningen; Netherlands Institute for Cultural Heritage, 21-27.

Bearman, G.H. and Spiro, S.I. (1996). Archaeological Applications of Advanced Imaging Techniques, The Biblical Archaeologist, 59(1): 56-66.

Chabries, D.M., Booras, S.W., \& Bearman, G. (2003). Imaging the past: Recent applications of multispectral imaging technology to deciphering manuscripts. Antiquity, 77(296), 359.
Clarkson, C. (1992). Rediscovering Parchment: the nature of the beast. The Paper Conservator, 16(1), 5-26.

Hardeberg, J.Y. (2001). Acquisition and Reproduction of Color Images: Colorimetric \& multispectral approaches, Dissertation.com

Landgrebe, D., \& Chen, C. (1999). Information Extraction Principles and Methods for Multispectral and Hyperspectral Image Data (pp. 3-38). River Edge, NJ: World Scientific Publishing Company.

Larsen, R. (2007). Introduction to damage and damage assessment of parchment. In Improved damage assessment of parchment (IDAP): assessment, data collection and sharing of knowledge. European Commission, DirectorateGeneral for Environment, 17-22.

Reed, R. (1972). Ancient skins, parchments and leathers, London; New York: Seminar Press.

Salerno, E., Tonazzini, A., \& Bedini, L. (2007). Digital image analysis to enhance underwritten text in the Archimedes palimpsest. International Journal on Document Analysis and Recognition, 9(2), 79-87.

Součková, M. (2007). Characterisation of microbial parchment damage based on the assessment of microbiologically accelerated aged parchments. In Improved damage assessment of parchment (IDAP): assessment, data collection and sharing of knowledge. European Comm., Directorate-General for Environment, pp. 31-35.

Tanner, S., \& Bearman, G. (2009). Digitizing the Dead Sea Scrolls. Proceedings of the IS\&T Archiving Conference (pp. 119-123).

Terras, M., \& Robertson, P. (2006). Image to Interpretation: An Intelligent System to Aid Historians in Reading the Vindolanda Texts ( $p$. 252). Oxford: Oxford University Press.

Vane, G., \& Goetz, A. F. H. (1988). Terrestrial imaging spectroscopy. Remote Sensing of Environment, 24(1), 1-29.

Vest, M., Jacobsen, J. \& Larsen, R. (2007). Accelerated ageing: effect of heat and relative humidity. In Improved damage assessment of parchment (IDAP): assessment, data collection and sharing of knowledge. European Commission, Directorate-General for Research, Directorate I-Environment, 67-68. 